\title{
Robust Parametric Macromodeling Using Multivariate Orthonormal Vector Fitting
}

\author{
Dirk Deschrijver, Tom Dhaene, Senior Member, IEEE, and Daniël De Zutter, Fellow, IEEE
}

\begin{abstract}
A robust multivariate extension of the orthonormal vector fitting technique is introduced for rational parametric macromodeling of highly dynamic responses in the frequency domain. The technique is applicable to data that is sparse or dense, deterministic or a bit noisy, and grid-based or scattered in the design space. For a specified geometrical parameter combination, a SPICE equivalent model can be calculated.
\end{abstract}

Index Terms-Least squares, parametric macromodels, rational functions, surface approximation, vector fitting.

\section{INTRODUCTION}

A CCURATE parametric macromodeling is becoming increasingly important for the design, study, and optimization of microwave structures. Parametric macromodels approximate the complex electromagnetic (EM) behavior of high-speed multiport systems at the input and output ports in the frequency domain. This behavior is typically characterized by the frequency and several geometrical parameters that describe physical properties of the structure, such as metallizations or substrate parameters. Such models are frequently used for efficient design space exploration, design optimization, and sensitivity analysis. Unfortunately, the calculation of models that exhibit sufficient accuracy is not a trivial task.

In the past, several multivariate modeling techniques were presented to tackle this problem. Artificial neural networks (ANNs) are a commonly used approach to calculate parametric models [1], [2]. Despite their ability to handle highly nonlinear behavior, it is hard to find a good topology, and large training sets may be required. Lamecki et al. applied the multivariate Cauchy method to calculate a parametric model by solving a set of interpolatory conditions [3]. It was found that the use of orthogonal Chebyshev polynomials or a parameter space division is required to avoid numerical ill-conditioning. Lehmensiek and Meyer avoid these numerical instabilities by using a multivariate extension of Thiele-type continued fractions as a rational interpolant [4], [5]. The continued fraction is recursively expanded by selecting additional data samples until a predefined accuracy level is reached. Despite its computational efficiency,

Manuscript received October 18, 2007; revised March 20, 2008. First published May 30, 2008; last published July 9, 2008 (projected). This work was supported by the Fund for Scientific Research in Flanders (FWO-Vlaanderen).

D. Deschrijver and D. De Zutter are with the Department of Information Technology (INTEC), Ghent University-Interdisciplinary Institute for Broadband Technology (IBBT), B-9000 Gent, Belgium.

T. Dhaene is with the Department of Information Technology (INTEC), Ghent University-Interdisciplinary Institute for Broadband Technology (IBBT), 9000 Gent, Belgium, and also with the Department of Mathematics and Computer Science, University of Antwerp, 2000 Antwerp, Belgium.

Digital Object Identifier 10.1109/TMTT.2008.924346 the technique cannot be applied if the data samples are contaminated with noise. De Geest et al. introduced multidimensional adaptive parameter sampling (MAPS) [6], which represents the multivariate model as a linear combination of multinomial basis functions with frequency-dependent coefficients. Even though the approach can provide accurate models, it was found that the combined rational-multinomial representation of the model can be somewhat restrictive if the data exhibits a large dynamic variation. The use of radial basis functions [7] and Kriging [8] has also been considered, but it was found that these methods provide less favorable results since they lack a theoretical connection with the physical problem at hand [9].

The parametric macromodeling technique that is presented in this paper is a robust multivariate extension of the vector fitting methodology [10]. It combines the use of an iterative least squares estimator [11], and orthonormal rational functions, which are based on a prescribed set of poles [12]. The approach allows the modeling of data sets, which are sparse or dense, and deterministic or noisy, using data samples, which are grid-based or scattered in the design space. It can also be applied to fit the entire scattering matrix of a structure using a common polebehavior, which increases the efficiency of time-domain convolutions. Based on a fixed choice of geometrical parameters, the multivariate model can easily be realized as an equivalent SPICE circuit [13]. Although the algorithm is applied to EM modeling problems in this paper, the basic concepts can be generalized to several other modeling problems in the field of computer-aided design.

\section{GOAL Statement}

The goal of the identification algorithm is to find a multivariate rational function $R(s, \vec{g})$, which approximates a large set of $K+1$ data samples $\left\{(s, \vec{g})_{k}, H(s, \vec{g})_{k}\right\}_{k=0}^{K}$ in a least squares sense. These data samples are usually $S$-parameters, which depend on a complex frequency $s=j \omega$, and several geometrical parameters $\vec{g}=\left\{g^{(n)}\right\}_{n=1}^{N}$. The geometrical parameters are design variables, which describe, e.g., the metallizations in an EM circuit (such as lengths, widths, etc.) or the substrate parameters (like thickness, dielectric constant, losses, etc.). The data samples are often deterministic, or contaminated by a small amount of measurement or simulation noise. In addition, the samples can be located on a grid or scattered in the design space. A generalization of the technique is required to fit the lower triangular part of the scattering matrix ( $Q$ elements) using a common pole behavior. By fixing the geometrical parameters of the multivariate model, a univariate model is obtained that can be realized as an equivalent SPICE circuit. 


\section{MODEL REPRESENTATION}

The multivariate rational model $R(s, \vec{g})$ is represented as the ratio of a numerator $N(s, \vec{g})$ and denominator $D(s, \vec{g})$

$$
R(s, \vec{g})=\frac{N(s, \vec{g})}{D(s, \vec{g})}=\frac{\sum_{p=0}^{P} \sum_{\vec{v} \in V} c_{p \vec{v}} \phi_{p}(s) \varphi_{\vec{v}}(\vec{g})}{\sum_{p=0}^{P} \sum_{\vec{v} \in V} \tilde{c}_{p \vec{v}} \phi_{p}(s) \varphi_{\vec{v}}(\vec{g})}
$$

where $s$ represents the complex frequency variable, and $P$ is the predefined maximal order of the frequency-dependent basis functions $\phi_{p}(s)$. The basis functions $\varphi_{\vec{v}}(\vec{g})$ are multivariate basis functions, which depend on a real parameter vector $\vec{g}=\left\{g^{(n)}\right\}_{n=1}^{N}$, containing $N$ real geometrical parameters. Such a basis function is defined as the product of $N$ univariate basis functions whose order is denoted by subindices $v_{n}$

$$
\varphi_{\vec{v}}(\vec{g})=\varphi_{v_{1}}\left(g^{(1)}\right) \cdots \varphi_{v_{N}}\left(g^{(N)}\right) \quad \forall \vec{v}=\left(v_{1}, \ldots, v_{N}\right) \in V .
$$

$V=\left\{\left(v_{1}, \ldots, v_{N}\right) \mid\left\{0 \leq v_{n} \leq V_{n}\right\}_{n=1}^{N}\right\}$ is a set that contains all distinct tuples $\vec{v}$ with nonnegative multiindices, while $V_{n}$ represents the predefined maximal order of the univariate basis functions $\varphi\left(g^{(n)}\right)$. Therefore, it follows that

$$
\begin{aligned}
& N(s, \vec{g}) \\
& \quad=\sum_{p=0}^{P} \sum_{v_{1}=0}^{V_{1}} \cdots \sum_{v_{N}=0}^{V_{N}} c_{p \vec{v}} \phi_{p}(s) \varphi_{v_{1}}\left(g^{(1)}\right) \cdots \varphi_{v_{N}}\left(g^{(N)}\right) \\
& D(s, \vec{g}) \\
& \quad=\sum_{p=0}^{P} \sum_{v_{1}=0}^{V_{1}} \cdots \sum_{v_{N}=0}^{V_{N}} \tilde{c}_{p \vec{v}} \phi_{p}(s) \varphi_{v_{1}}\left(g^{(1)}\right) \cdots \varphi_{v_{N}}\left(g^{(N)}\right) .
\end{aligned}
$$

\section{MODEL IDENTIFICATION}

The goal of the identification algorithm is to find the optimal values of the coefficients $c_{p \vec{v}}$ and $\tilde{c}_{p \vec{v}}$ in (1) such that the $L_{2}$-norm $\left\|R(s, \vec{g})_{k}-H(s, \vec{g})_{k}\right\|_{2}$ over all data samples is minimized. A linear approximation to this nonlinear optimization problem is obtained by using an iterative procedure called the Sanathanan-Koerner iteration [11], [14]. In the first iteration step $(t=0)$, Levi's cost function is minimized to obtain an initial (biased) estimate of the model parameters [15]

$$
\min _{c_{p \vec{v}}, \tilde{c}_{p \vec{v}}} \sum_{k=0}^{K}\left|N^{(0)}(s, \vec{g})_{k}-D^{(0)}(s, \vec{g})_{k} H(s, \vec{g})_{k}\right|^{2} \text {. }
$$

Based on the initial estimate of the coefficients $\tilde{c}_{p \vec{v}}$, the denominator $D^{(0)}(s, \vec{g})$ can be used as an inverse weighting to the system equations. By repeating this process iteratively $(t=$ $1, \ldots, T)$, updated values of the coefficients $c_{p \vec{v}}^{(t)}$ and $\tilde{c}_{p \vec{v}}^{(t)}$ can be derived by minimizing the following cost function [16]:

$$
\min _{c_{p \vec{v}}^{(t)}, \tilde{c}_{p \vec{v}}^{(t)}} \sum_{k=0}^{K}\left|\frac{N^{(t)}(s, \vec{g})_{k}}{D^{(t-1)}(s, \vec{g})_{k}}-\frac{D^{(t)}(s, \vec{g})_{k}}{D^{(t-1)}(s, \vec{g})_{k}} H(s, \vec{g})_{k}\right|^{2} .
$$

The trivial null solution can typically be avoided by fixing one coefficient (e.g., the constant term of the denominator) to unity. This can be done without loss of generality since both the numerator and denominator can be divided by the same constant value. In this implementation, the null solution is avoided by introducing a more relaxed nontriviality constraint as an additional row in the system matrix. The constraint imposes that the sum of the denominator samples approaches a nonzero value without fixing any of its coefficients

$$
\Re e\left(\sum_{k=0}^{K} \frac{D^{(t)}(s, \vec{g})_{k}}{D^{(t-1)}(s, \vec{g})_{k}}\right)=K+1 .
$$

This equation is given a weighting in relation to the size of the data with weight $=\|H(s, \vec{g})\| /(K+1)$. It was shown in [17] that usage of this constraint often leads to more accurate results, especially when the data samples are contaminated with noise. To ensure that the model coefficients $c_{p \vec{v}}^{(t)}, \tilde{c}_{p \vec{v}}^{(t)}$ are real, each equation is split in the real and imaginary parts. Therefore, the eventual cost function that needs to be minimized at iteration step $t$ is defined as follows:

$$
\min _{c_{p \vec{v}}^{(t)}, \tilde{c}_{p \vec{v}}^{(t)}} \sum_{k=0}^{K}\left(\left|\Re e\left(e_{1}^{(t)}\right)\right|^{2}+\left|\Im m\left(e_{1}^{(t)}\right)\right|^{2}\right)+\left|\Re e\left(e_{2}^{(t)}\right)\right|^{2}
$$

provided that $e_{1}^{(t)}$ and $e_{2}^{(t)}$ are defined as

$$
\begin{aligned}
e_{1}^{(t)} & =\frac{N^{(t)}(s, \vec{g})_{k}}{D^{(t-1)}(s, \vec{g})_{k}}-\frac{D^{(t)}(s, \vec{g})_{k}}{D^{(t-1)}(s, \vec{g})_{k}} H(s, \vec{g})_{k} \\
e_{2}^{(t)} & =\frac{\|H(s, \vec{g})\|}{(K+1)}\left(\sum_{k=0}^{K} \frac{D^{(t)}(s, \vec{g})_{k}}{D^{(t-1)}(s, \vec{g})_{k}}\right)-\|H(s, \vec{g})\| .
\end{aligned}
$$

In order to improve the numerical accuracy of the results, each column of the system equations is scaled to unit length.

\section{CHOICE OF BASIS FUnCTIONS}

\section{A. Frequency-Dependent Basis Functions}

The frequency-dependent behavior of a complex structure is often highly dynamic due to the occurrence of resonances and coupling effects. Therefore, a set of Muntz-Laguerre orthonormal rational basis functions $\phi_{p}(s, \vec{a})$ is chosen, which are based on a prescribed set of stable poles $\vec{a}=\left\{-a_{p}\right\}_{p=1}^{P}$ provided that $\phi_{0}(s)=1$ [12], [18]. If $-a_{p}$ is a real pole, then these basis functions are defined by the following expression:

$$
\phi_{p}(s, \vec{a})=\frac{\sqrt{2 \Re e\left(a_{p}\right)}}{s+a_{p}}\left(\prod_{i=1}^{p-1} \frac{s-a_{i}^{*}}{s+a_{i}}\right) .
$$

If $-a_{p}$ and $-a_{p+1}$ constitute a complex conjugate pair of poles ( such that $-a_{p+1}=-a_{p}^{*}$ ), then a linear combination of the two corresponding basis functions is formed as follows:

$$
\begin{aligned}
\phi_{p}(s, \vec{a}) & =\frac{\sqrt{2 \Re e\left(a_{p}\right)}\left(s-\left|a_{p}\right|\right)}{\left(s+a_{p}\right)\left(s+a_{p+1}\right)}\left(\prod_{i=1}^{p-1} \frac{s-a_{i}^{*}}{s+a_{i}}\right) \\
\phi_{p+1}(s, \vec{a}) & =\frac{\sqrt{2 \Re e\left(a_{p}\right)}\left(s+\left|a_{p}\right|\right)}{\left(s+a_{p}\right)\left(s+a_{p+1}\right)}\left(\prod_{i=1}^{p-1} \frac{s-a_{i}^{*}}{s+a_{i}}\right) .
\end{aligned}
$$

It was shown in [19] and [20] that the use of orthonormal rational basis functions leads to a numerically more robust procedure than the use of a partial fraction basis, especially if the initial pole location is specified in a suboptimal way. 


\section{B. Geometrical Parameter-Based Basis Functions}

1) Polynomial Basis Functions: It is observed in many practical applications that the model response varies smoothly if a geometrical parameter is adjusted. This means that the geometrical basis functions $\varphi_{\vec{v}}(\vec{g})$ usually have relatively low orders. Therefore, a suitable choice of basis functions for these parameters is a set of polynomial basis functions, which depend on a real variable $g^{(n)}$ having real coefficients. The most obvious choice is a classical power series basis. In that case, the multivariate basis functions are defined as

$$
\varphi_{\vec{v}}(\vec{g})=\left(g^{(1)}\right)^{v_{1}} \cdots\left(g^{(N)}\right)^{v_{N}} \quad \forall \vec{v}=\left(v_{1}, \ldots, v_{N}\right) \in V
$$

In some cases where ill-conditioning may arise (e.g., due to a highly dynamic behavior in the parameter ranges), orthogonal Chebyshev polynomials of the first kind can be applied [21]. If the parameter ranges are appropriately scaled, it is well known that they can improve the numerical conditioning of the system equations [22]. Even though polynomial basis functions are used, the model representation (1) remains completely rational both in terms of the frequency and geometrical parameters. Perhaps it should be emphasized that the choice of polynomial basis functions does not impose a polynomial variation of the model in terms of the geometrical parameters. However, a known drawback of orthogonal polynomials is that the evaluation of their three-term recurrence relation is slow and may require a prohibitive amount of computation time.

2) Rational Basis Functions: An alternative solution to deal with high model orders is obtained by using a set of rational basis functions, which are formulated as a function of $j g^{(n)}$. These basis functions are based on a prescribed set of starting poles $\vec{b}^{(n)}=\left\{-b_{v_{n}}^{(n)}\right\}_{v_{n}=1}^{V_{n}}$, which is computed for each geometrical parameter $g^{(n)}$ individually. The poles are chosen as complex pairs $b_{v_{n+1}}^{(n)}=-\left(b_{v_{n}}^{(n)}\right)^{*}$, which have real parts of opposite sign. Using these poles, it is possible to analytically enforce that the response of the basis functions has a zero-phase angle. This ensures that the corresponding basis functions $\varphi_{v_{n}}\left(g^{(n)}, \vec{b}^{(n)}\right)$ and $\varphi_{v_{n}+1}\left(g^{(n)}, \vec{b}^{(n)}\right)$ are real by construction. The basis functions are, therefore, defined as

$$
\begin{aligned}
\varphi_{v_{n}}\left(g^{(n)}, \vec{b}^{(n)}\right) & =\left(j g^{(n)}+b_{v_{n}}^{(n)}\right)^{-1}-\left(j g^{(n)}-\left(b_{v_{n}}^{(n)}\right)^{*}\right)^{-1} \\
& =\frac{2 \Re e\left(b_{v_{n}}^{(n)}\right)}{\left(g^{(n)}\right)^{2}+2 \Im m\left(b_{v_{n}}^{(n)}\right) g^{(n)}+\left|b_{v_{n}}^{(n)}\right|^{2}}
\end{aligned}
$$

and

$$
\begin{aligned}
\varphi_{v_{n}+1}\left(g^{(n)}, \vec{b}^{(n)}\right) \\
=j\left(j g^{(n)}+b_{v_{n}}^{(n)}\right)^{-1}+j\left(j g^{(n)}-\left(b_{v_{n}}^{(n)}\right)^{*}\right)^{-1} \\
=\frac{2 g^{(n)}+2 \Im m\left(b_{v_{n}}^{(n)}\right)}{\left(g^{(n)}\right)^{2}+2 \Im m\left(b_{v_{n}}^{(n)}\right) g^{(n)}+\left|b_{v_{n}}^{(n)}\right|^{2}}
\end{aligned}
$$

with $\varphi_{0}\left(g^{(n)}, \vec{b}^{(n)}\right)=1$ [23]. Complex conjugacy of the poles $\vec{b}^{(n)}$ is not enforced here because there is no symmetric relationship between the model response for $g^{(n)}$ and $-g^{(n)}$.

\section{Choice of Starting Poles}

\section{A. Frequency}

The starting poles of the frequency-dependent basis functions $\phi_{p}(s, \vec{a})$ and $\phi_{p+1}(s, \vec{a})$ are chosen as stable complex conjugate pairs $\left(-a_{p+1}=-a_{p}{ }^{*}\right)$, which have small real parts $\left(\Re e\left(-\alpha_{p}\right)<0\right)$, and their imaginary parts $\beta_{p}$ linearly spaced over the frequency range of interest such that

$$
\begin{aligned}
-a_{p} & =-\alpha_{p}+j \beta_{p} \quad-a_{p+1}=-\alpha_{p}-j \beta_{p} \\
\left\{\alpha_{p}\right\} & =0.01\left\{\beta_{p}\right\} .
\end{aligned}
$$

It was shown in [10] that this distribution of the poles reduces the probability that poles must be relocated over long distances and, therefore, avoids that the Sanathanan-Koerner weighting exhibits a large dynamic variation, which breaks down the numerical conditioning of the system equations [20].

\section{B. Geometrical Parameters}

The starting poles of the geometrical basis functions $\varphi_{v_{n}}\left(g^{(n)}, \vec{b}^{(n)}\right)$ and $\varphi_{v_{n}+1}\left(g^{(n)}, \vec{b}^{(n)}\right)$ are chosen as complex pairs, which have small real parts of opposite sign $\left(-\alpha_{v_{n}}, \alpha_{v_{n}}\right)$, and their imaginary parts $\beta_{v_{n}}$ linearly spaced over the geometrical parameter range of interest such that

$$
\begin{aligned}
& -b_{v_{n}}^{(n)}=-\alpha_{v_{n}}+j \beta_{v_{n}}-b_{v_{n}+1}^{(n)}=\alpha_{v_{n}}+j \beta_{v_{n}} \\
& \left\{\alpha_{v_{n}}\right\}=0.01\left\{\beta_{v_{n}}\right\} .
\end{aligned}
$$

This choice of starting poles ensures that the geometrical basis functions have a zero-phase angle since $b_{v_{n}+1}^{(n)}=-\left(b_{v_{n}}^{(n)}\right)^{*}$.

\section{Multivariate Model to SPICE}

Given a fixed choice of values for the geometrical parameters $\vec{g}_{m}=\left(g_{m}^{(1)}, \ldots, g_{m}^{(N)}\right)$, the multivariate rational model (1) reduces to a univariate frequency-dependent transfer function

$$
\begin{aligned}
R\left(s, \vec{g}_{m}\right) & =\frac{\sum_{p=0}^{P} \sum_{\vec{v} \in V} c_{p \vec{v}}^{(T)} \phi_{p}(s) \varphi_{\vec{v}}\left(\vec{g}_{m}\right)}{\sum_{p=0}^{P} \sum_{\vec{v} \in V} \tilde{c}_{p \vec{v}}^{(T)} \phi_{p}(s) \varphi_{\vec{v}}\left(\vec{g}_{m}\right)} \\
& =\frac{\sum_{p=0}^{P} \gamma_{p} \phi_{p}(s)}{\sum_{p=0}^{P} \tilde{\gamma}_{p} \phi_{p}(s)}=\frac{N\left(s, \vec{g}_{m}\right)}{D\left(s, \vec{g}_{m}\right)}
\end{aligned}
$$

where $\gamma_{p}=\sum_{\vec{v} \in V} c_{p \vec{v}}^{(T)} \varphi_{\vec{v}}\left(\vec{g}_{m}\right)$ and $\tilde{\gamma}_{p}=\sum_{\vec{v} \in V} \tilde{c}_{p \vec{v}}^{(T)} \varphi_{\vec{v}}\left(\vec{g}_{m}\right)$ are real coefficients. Since the prescribed poles $\vec{a}$ of $N\left(s, \vec{g}_{m}\right)$ and $D\left(s, \vec{g}_{m}\right)$ cancel out, it is clear that the poles of $R\left(s, \vec{g}_{m}\right)$ are actually the zeros of $D\left(s, \vec{g}_{m}\right)$. By constructing the real minimal state space realization $(A, B, C, D)$ of $D\left(s, \vec{g}_{m}\right)$,

$$
\begin{aligned}
s X(s) & =A X(s)+B U(s) \\
Y(s) & =C X(s)+D U(s)
\end{aligned}
$$

the poles of $R\left(s, \vec{g}_{m}\right)$ are directly identified by solving the eigenvalues of $A-B D^{-1} C$. More details on this procedure are described in [19]. For the convenience of the reader, a short summary of the key results is reported in the Appendix. Once the poles $\left\{-\theta_{p}\right\}_{p=1}^{P}$ of a univariate transfer function 
$R\left(s, \vec{g}_{m}\right)$ are known, unstable poles can be flipped into the left half-plane and the corresponding residues are found by solving a linear problem. This univariate pole-residue model can then be subjected to standard passivity enforcement techniques (see [24] and [25]), and the realization of an equivalent circuit is straightforward (see [24] and [13]). The construction of multivariate macromodels, which are stable and passive by construction, will be reported in a future paper [26].

\section{Multiple-InPut Multiple-OutPut (MIMO) FITTING USING COMMON POLES}

In some cases, it is desired to fit multiport systems such that the pole-behavior as a function of the geometrical parameters $\vec{g}$ is identical. This pole behavior is characterized by pole trajectories for bivariate rational models or pole hyperplanes for higher dimensional systems. The goal of this approach is that the univariate transfer function of each element (for a given geometrical parameter configuration $\vec{g}_{m}$ ) shares a common set of poles, which increases the efficiency of the time-domain convolutions. Therefore, a vector of $Q$ elements is modeled by rational functions, which share a common denominator $D^{(t)}(s, \vec{g})_{k}$ having coefficients $\tilde{c}_{p \vec{v}}^{(t)}$ and a distinct numerator $N_{q}^{(t)}(s, \vec{g})_{k}$ having coefficients $\tilde{c}_{q, p \vec{v}}^{(t)}$ (for $1 \leq q \leq Q$ ). These coefficients are estimated in such way that the following cost function is minimized at each iteration step $t$ :

$$
\min _{c_{q, p \vec{v}}^{(t)}, \tilde{c}_{p \vec{v}}^{(t)}} \sum_{q=1}^{Q} \sum_{k=0}^{K}\left(\left|\Re e\left(e_{q, 1}^{(t)}\right)\right|^{2}+\left|\Im m\left(e_{q, 1}^{(t)}\right)\right|^{2}\right)+\left|\Re e\left(e_{2}^{(t)}\right)\right|^{2}
$$

provided that $e_{q, 1}^{(t)}$ and $e_{2}^{(t)}$ are defined as

$$
\begin{aligned}
e_{q, 1}^{(t)} & =\frac{N_{q}^{(t)}(s, \vec{g})_{k}}{D^{(t-1)}(s, \vec{g})_{k}}-\frac{D^{(t)}(s, \vec{g})_{k}}{D^{(t-1)}(s, \vec{g})_{k}} H_{q}(s, \vec{g})_{k} \\
e_{2}^{(t)} & =\frac{\|H(s, \vec{g})\|}{(K+1)} \sum_{k=0}^{K} \frac{D^{(t)}(s, \vec{g})_{k}}{D^{(t-1)}(s, \vec{g})_{k}}-\|H(s, \vec{g})\| .
\end{aligned}
$$

It is observed that the system equations corresponding to (23) may become very large. To obtain a significant speed up in terms of computation time and memory requirements, a fast implementation of the vector fitting technique can be used, which exploits the sparsity of the system equations [27].

\section{2-D EXAMPLE: IRIS IN RECTANGULAR WAVEGUIDE}

The reflection coefficient $S_{11}$ and transmission coefficient $S_{21}$ of equidistantly placed 2-D irises in a rectangular waveguide are computed using the mode-matching method [3], as shown in Fig. 1. Based on a dense set of $S$-parameter data samples, a multivariate macromodel is calculated as a function of a varying iris height $h$ with $h \in(3.5-9 \mathrm{~mm})$ over the frequency range (12-18 GHz).

Initially, 34 complex conjugate starting poles are selected to model the frequency behavior, and 18 complex starting poles are chosen for the geometrical parameter, as described in Section VI. In order to have an accurate model, an overall absolute fitting error of $-60 \mathrm{~dB}$ or smaller is desired, which corresponds to approximately three significant digits. The

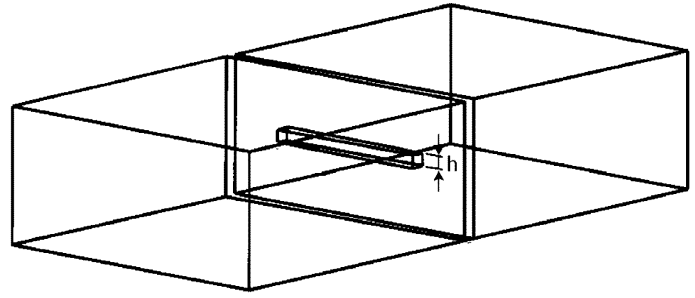

Fig. 1. Iris in rectangular waveguide [4].

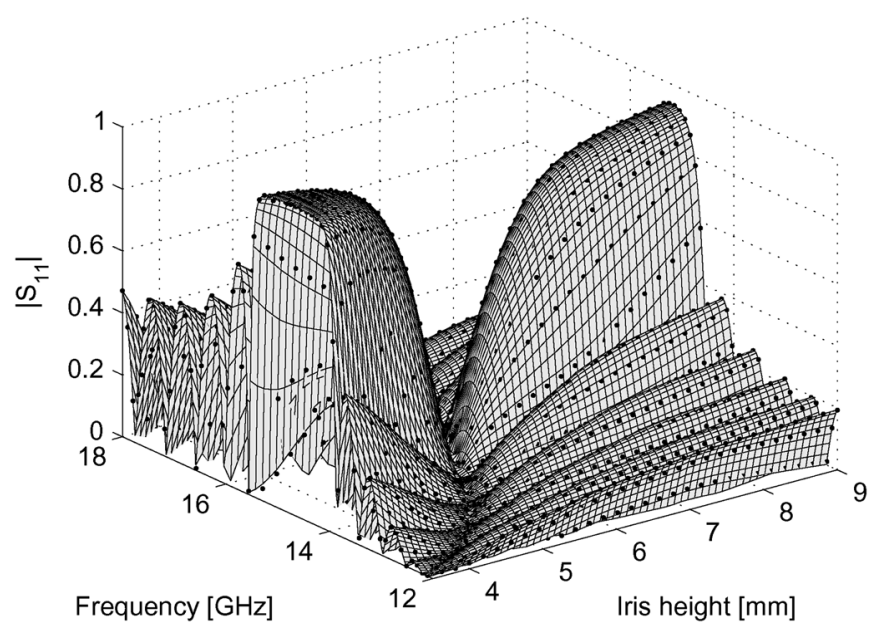

Fig. 2. Reflection coefficient $S_{11}$ of bivariate model.

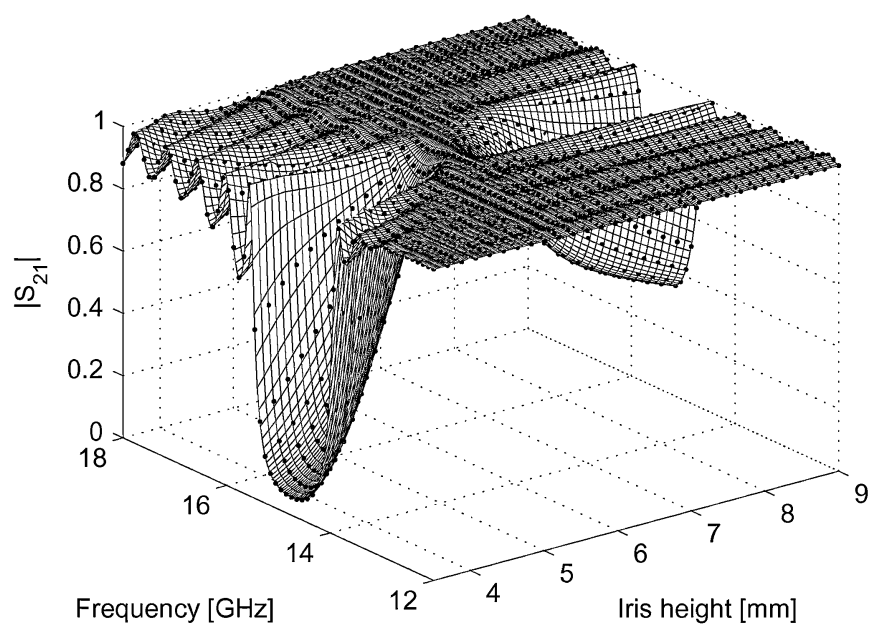

Fig. 3. Transmission coefficient $S_{21}$ of bivariate model.

proposed algorithm is applied to the data samples, and an accurate macromodel is calculated in only four iterations. The approximation error of $S_{11}$ and $S_{21}$ in the selected data samples corresponds to $-68.16 \mathrm{~dB}\left(S_{11}\right)$ and $-68.91 \mathrm{~dB}\left(S_{21}\right)$, which is quite satisfactory.

Figs. 2 and 3 show the response of the multivariate rational model when evaluated on a grid that is much denser than the initial sampling. The smoothness of the approximation surface indicates that a good overall approximation is obtained, also in between the selected data samples. An overall assessment of the model quality can then be acquired by computing a set of validation data samples using the mode-matching method. As 


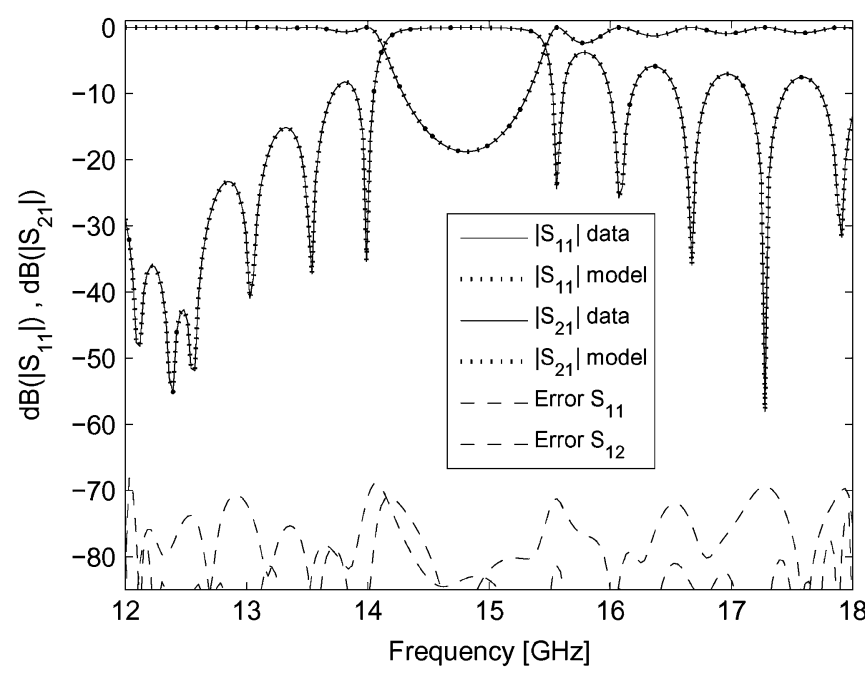

Fig. 4. Model validation: frequency response for $h=3.9 \mathrm{~mm}$.

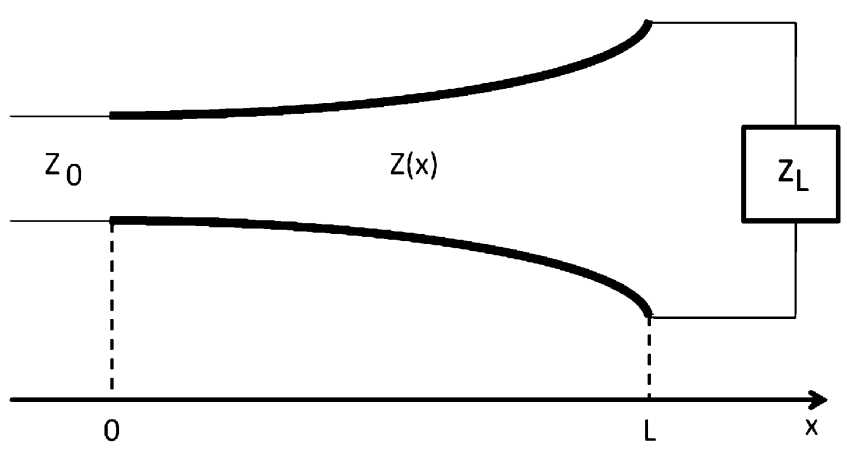

Fig. 5. Exponential tapered microstrip transmission line [29].

an illustration, the univariate frequency response of the model is calculated for $h=3.9 \mathrm{~mm}$, as shown in Fig. 4. Clearly, an good correspondence is observed between the interpolated response and 200 calculated validation samples.

\section{3-D EXAMPLE: TAPERED TRANSMISSION LINE}

The presented technique is used to model the reflection coefficient $S_{11}$ of a lossless exponential tapered transmission line [28], [29] that is terminated with a matched load, as shown in Fig. 5, where $Z_{0}=50 \Omega$ and $Z_{L}=100 \Omega$ represent the reference impedance and the load impedance, respectively.

A parametric macromodel is computed as a function of the varying relative dielectric constant $\varepsilon_{r} \in(3-5)$ and varying line length $L \in(1-10 \mathrm{~cm})$ over the frequency range $(1 \mathrm{kHz}-3 \mathrm{GHz})$. The initial data is computed over a grid of $10 \times 20 \times 30$ samples, and the corresponding number of poles is set to 4,6 , and 12 , respectively. Fig. 6 shows the frequency response of the trivariate structure for a fixed value of $\varepsilon_{r}=5$, while Fig. 7 shows the variation of the response for an increasing line length $L$. The proposed algorithm converges to accurate results in only two iterations. The calculation of the macromodel takes approximately $28 \mathrm{~s}$ on a standard desktop computer in MATLAB, and requires $87 \mathrm{MB}$ of RAM to store the least squares matrix in (6). The maximum absolute error of $S_{11}$ in the initial data samples corresponds to $-74.51 \mathrm{~dB}$. An overall assessment of the model quality is then acquired by computing a set of validation data

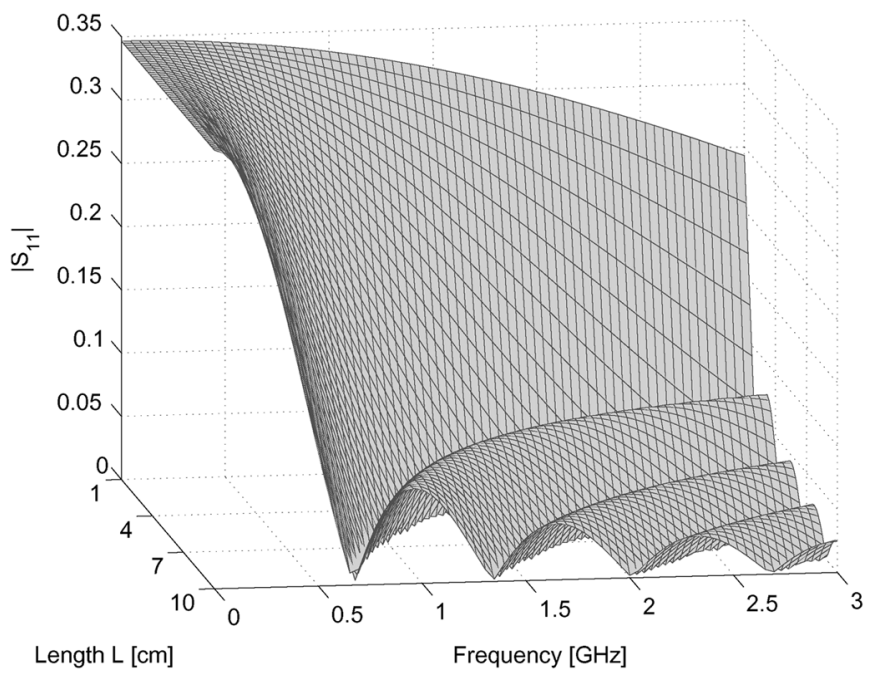

Fig. 6. Reflection coefficient $S_{11}$ for $\varepsilon_{r}=5$.

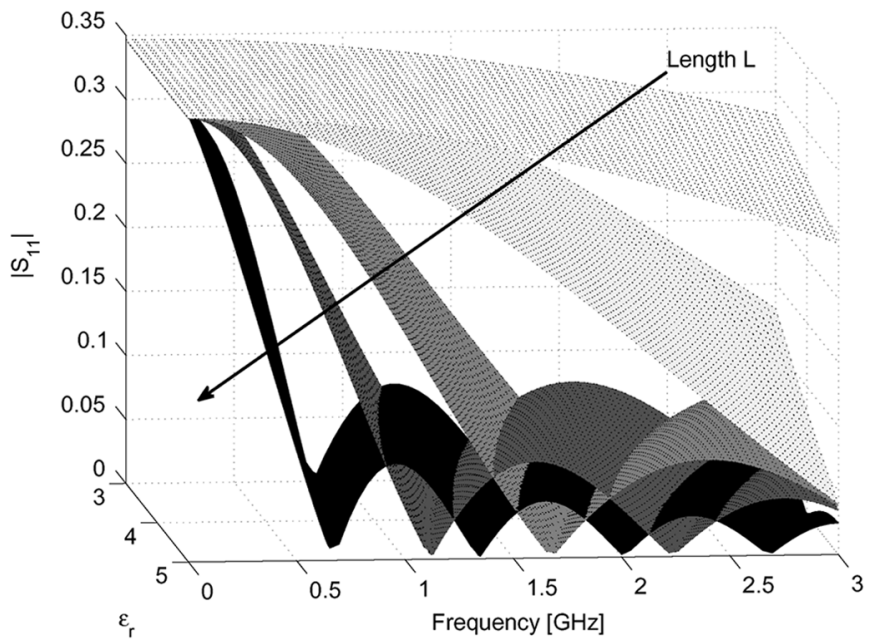

Fig. 7. Reflection coefficient $S_{11}$ for $L=1,2,4,6,10 \mathrm{~cm}$.

samples on a very dense grid of size $30 \times 50 \times 70$. The number of validation samples that corresponds to a certain absolute error is shown by a histogram in Fig. 8. It clearly shows that the computed macromodel has a good overall accuracy since the maximum absolute error over all the validation samples is bounded by $-65.72 \mathrm{~dB}$.

\section{Practical Aspects AND Limitations}

- Finding the least squares solution of (6) is computationally the most expensive part of the algorithm because the complexity scales cubically with the size of the problem. As the number of geometrical parameters increases, the required amount of data samples grows exponentially with the dimension. Even for a moderate amount of parameters, the memory storage requirements and overall computation time may become prohibitively large. In order to reduce the computational burden, adaptive sampling strategies, as shown in [6] and [3], can be applied.

- As most full-wave EM techniques [including the finite-difference time-domain (FDTD), finite-element 


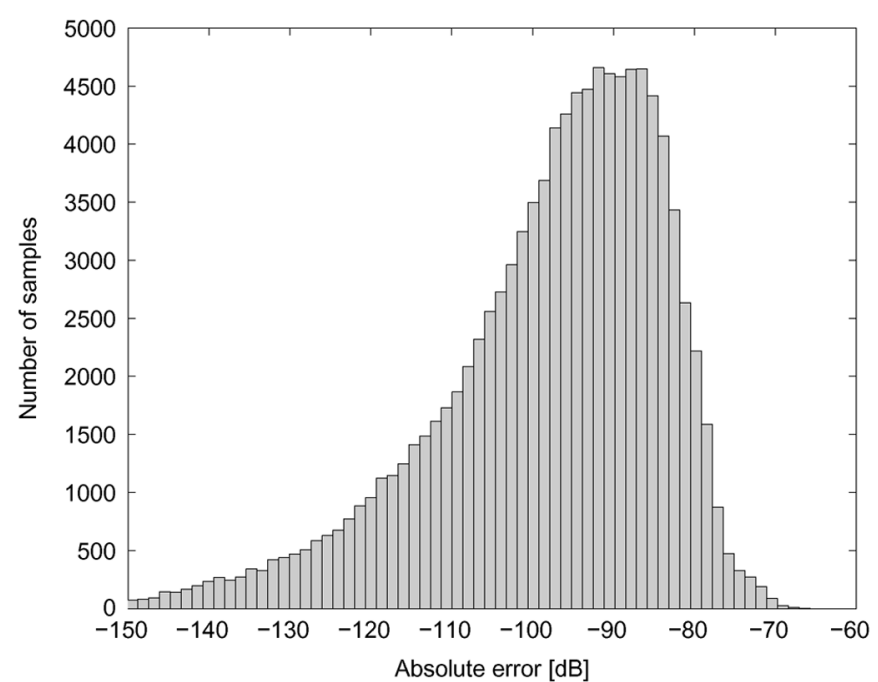

Fig. 8. Histogram: error distribution over 105000 validation samples.

method (FEM), and method of moments (MoM)] discretize the computational space, the computed simulation data can sometimes be polluted by discretization noise. This is caused by the fact that the relative grid size may vary in a nonsmooth way as a function of the geometrical parameters. The presented method can handle data with a sufficiently high signal-to-noise ratio because it is based on least squares fitting and relaxation. However, if the input data contains nonnegligible discontinuities, then the modeling error between the parametric macromodel and the data may increase locally. Such problems are inherent to the quality of the data and do not affect the validity of the parametric macromodeling approach.

\section{CONCLUSION}

A multivariate extension of the orthonormal vector fitting technique has been presented for the calculation of parametric macromodels from frequency-response data. The robustness of the proposed method has been obtained by combining the use of rational basis functions and an iterative weighting scheme. Several examples have illustrated that the approach is able to model highly dynamical responses with a good accuracy. Once the model has been calculated, it can efficiently be used for fast design space exploration, design optimization, and sensitivity analysis.

\section{APPENDIX}

The minimal continuous-time linear time-invariant (LTI) state-space realization

$$
\begin{aligned}
s X(s) & =A X(s)+B U(s) \\
Y(s) & =C X(s)+D U(s)
\end{aligned}
$$

of the denominator

$$
D\left(s, \vec{g}_{m}\right)=\sum_{p=0}^{P} \tilde{\gamma}_{p} \phi_{p}(s)
$$

is defined as

$$
\begin{aligned}
& A=\left[\begin{array}{ccccc}
-a_{1} & 0 & 0 & \ldots & 0 \\
2 \Re e\left(-a_{1}\right) & -a_{2} & 0 & \ldots & 0 \\
2 \Re e\left(-a_{1}\right) & 2 \Re e\left(-a_{2}\right) & -a_{3} & \ldots & 0 \\
2 \Re e\left(-a_{1}\right) & 2 \Re e\left(-a_{2}\right) & 2 \Re e\left(-a_{3}\right) & \ldots & 0 \\
\ldots & \ldots & \ldots & \ldots & \ldots \\
2 \Re e\left(-a_{1}\right) & 2 \Re e\left(-a_{2}\right) & 2 \Re e\left(-a_{3}\right) & \ldots & -a_{P}
\end{array}\right] \\
& B=\left[\begin{array}{llll}
1 & 1 & \cdots & 1
\end{array}\right]^{\mathrm{T}} \\
& C=\left[\begin{array}{c}
\tilde{\gamma}_{1} \sqrt{2 \Re e\left(a_{1}\right)} \\
\tilde{\gamma}_{2} \sqrt{2 \Re e\left(a_{2}\right)} \\
\ldots \\
\tilde{\gamma}_{P} \sqrt{2 \Re e\left(a_{P}\right)}
\end{array}\right]^{\mathrm{T}} \\
& D=\tilde{\gamma}_{0}
\end{aligned}
$$

provided that the poles $-a_{p}$ are real. If $-a_{p}$ and $-a_{p+1}$ constitute a complex conjugate pair of poles (i.e., $-a_{p+1}=-a_{p}^{*}$ ), then a real-valued realization is obtained by replacing

$$
\left(\begin{array}{cc}
-a_{p} & 0 \\
2 \Re e\left(-a_{p}\right) & -a_{p+1}
\end{array}\right)
$$

in the state matrix A by

$$
\left(\begin{array}{cc}
\Re e\left(-a_{p}\right) & \Re e\left(-a_{p}\right)-\left|a_{p}\right| \\
\Re e\left(-a_{p}\right)+\left|a_{p}\right| & \Re e\left(-a_{p}\right)
\end{array}\right) .
$$

\section{ACKNOWLEDGMENT}

The authors thank Dr. A. Lamecki and Prof. M. Mrozowski, both with the Polytechnical University of Gdansk, Gdansk, Poland, for providing the data of the rectangular waveguide, and F. Ferranti, Ghent University, Gent, Belgium, for providing the data of the exponential tapered transmission line.

\section{REFERENCES}

[1] A. H. Zaabab, Q. J. Zhang, and M. Nakhla, "A neural network modeling approach to circuit optimization and statistical design," IEEE Trans. Microw. Theory Tech., vol. 43, no. 6, pp. 1349-1558, Jun. 1995.

[2] L. Balewski and M. Mrozowski, "Creating neural models using an adaptive algorithm for optimal size of neural network and training set," in 15th Int. Microw., Radar, Wireless Commun. Conf., May 2004, vol. 2, pp. 543-546.

[3] A. Lamecki, P. Kozakowski, and M. Mrozowski, "Efficient implementation of the Cauchy method for automated CAD-model construction," IEEE Microw. Wireless Compon. Lett., vol. 13, no. 7, pp. 268-270, Jul. 2003.

[4] R. Lehmensiek and P. Meyer, "Creating accurate multivariate rational interpolation models of microwave circuits by using efficient adaptive sampling to minimize the number of computational electromagnetic analyses," IEEE Trans. Microw. Theory Tech., vol. 49, no. 8, pp. 1419-1430, Aug. 2001.

[5] R. Lehmensiek and P. Meyer, "Adaptive sampling applied to multivariate, multiple-output rational interpolation models with applications to microwave circuits," Int. J. RF Microw. Comput.-Aided Eng., vol. 12, no. 4, pp. 332-340, Jun. 2002. 
[6] J. De Geest, T. Dhaene, N. Faché, and D. De Zutter, "Adaptive CADmodel building algorithm for general planar microwave structures," IEEE Trans. Microw. Theory Tech., vol. 47, no. 9, pp. 1801-1809, Sep. 1999.

[7] A. Lamecki, P. Kozakowski, and M. Mrozowski, "CAD-model construction based on adaptive radial basis functions interpolation technique," in 15th Int. Microw., Radar, Wireless Commun. Conf., May 2004, vol. 3, pp. 799-802.

[8] J. P. C. Kleijnen and W. C. M. Van Beers, "Application driven sequential designs for simulation experiments: Kriging metamodels," J. Operation. Res. Soc., vol. 55, pp. 876-883, Dec. 2004.

[9] W. Hendrickx and T. Dhaene, "Meta-modelling of microwave devices with rational functions and radial basis functions," in 16th Int. Microw., Radar, Wireless Commun. Conf., May 2006, vol. 3, pp. 973-976.

[10] B. Gustavsen and A. Semlyen, "Rational approximation of frequency domain responses by vector fitting," IEEE Trans. Power Del., vol. 14, no. 3, pp. 1052-1061, Jul. 1999.

[11] C. Sanathanan and J. Koerner, "Transfer function synthesis as a ratio of two complex polynomials," IEEE Trans. Autom. Control, vol. AC-8, no. 1 , pp. 56-58, Jan. 1963.

[12] P. S. C. Heuberger, P. M. J. Van Den Hof, and B. Wahlberg, Modelling and Identification with Rational Orthogonal Basis Functions. London, U.K.: Springer-Verlag, 2005.

[13] G. Antonini, "SPICE equivalent circuits of frequency-domain responses," IEEE Trans. Electromagn. Compat., vol. 45, no. 3, pp. 502-512, Aug. 2003.

[14] K. Steiglitz and L. E. McBride, "A technique for the identification of linear systems," IEEE Trans. Autom. Control, vol. AC-10, no. 4, pp. 461-464, Oct. 1965.

[15] E. C. Levi, "Complex curve fitting," IRE Trans. Autom. Control, vol. AC-4, no. 1, pp. 37-44, Jan. 1959.

[16] W. Hendrickx, D. Deschrijver, and T. Dhaene, "Some remarks on the vector fitting iteration," in Progress in Industrial Mathematics at ECMI 2004, ser. Math. Indus. Berlin, Germany: Springer-Verlag, 2006, vol. 8, pp. 134-138.

[17] B. Gustavsen, "Improving the pole relocating properties of vector fitting," IEEE Trans. Power Del., vol. 21, no. 3, pp. 1587-1592, Jul. 2006.

[18] L. Knockaert, "On orthonormal Muntz-Laguerre filters," IEEE Trans. Signal Process., vol. 49, no. 4, pp. 790-793, Apr. 2001.

[19] D. Deschrijver, B. Haegeman, and T. Dhaene, "Orthonormal vector fitting: A robust macromodeling tool for rational approximation of frequency domain responses," IEEE Trans. Adv. Packag., vol. 30, no. 2, pp. 216-225, May 2007.

[20] D. Deschrijver, B. Gustavsen, and T. Dhaene, "Advancements in iterative methods for rational approximation in the frequency domain," IEEE Trans. Power Del., vol. 22, no. 3, pp. 1633-1642, Jul. 2007.

[21] M. Abramowitz and I. A. Stegun, "Orthogonal polynomials," in Handbook of Mathematical Functions with Formula's, Graphs, and Mathematical Tables, 9th ed. New York: Dover, 1972.

[22] R. Gao, Y. S. Mekonnen, W. T. Beyene, and J. E. Schutt-Aine, "Black-box modeling of passive systems by rational function approximation," IEEE Trans. Adv. Packag., vol. 28, no. 2, pp. 209-215, May 2005.

[23] L. De Tommasi and B. Gustavsen, "Low order transmission line modeling by modal decomposition and minimum phase shift fitting," in IEEE Signal Propag. Interconnects Workshop, Berlin, Germany, May 2006, pp. 89-92.

[24] A. Lamecki and M. Mrozowski, "Equivalent SPICE circuits with guaranteed passivity from nonpassive models," IEEE Trans. Microw. Theory Tech., vol. 55, no. 3, pp. 526-532, Mar. 2007.

[25] S. Grivet-Talocia, "Passivity enforcement via perturbation of Hamiltonian matrices," IEEE Trans. Circuits Syst. I, Reg. Papers, vol. 51, no. 9, pp. 1755-1769, Sep. 2004.

[26] D. Deschrijver and T. Dhaene, "Stability and passivity enforcement of parametric macromodels in time and frequency domain," IEEE Trans. Microw. Theory Tech., Feb. 2008, submitted for publication.

[27] D. Deschrijver, M. Mrozowski, T. Dhaene, and D. De Zutter, "Macromodeling of multiport systems using a fast implementation of the vector fitting method," IEEE Microw. Wireless Compon. Lett., vol. 18, no. 6 , Jun. 2008 , to be published.
[28] D. M. Pozar, Microwave Engineering, 2nd ed. New York: Wiley, 1998.

[29] M. Hadi Eghlidi, K. Mehrany, and B. Rashidian, "Analytical approach for analysis of nonuniform lossy/lossless transmission lines and tapered microstrips," IEEE Trans. Microw. Theory Tech., vol. 54, no. 12, pp. 4122-4129, Dec. 2006.

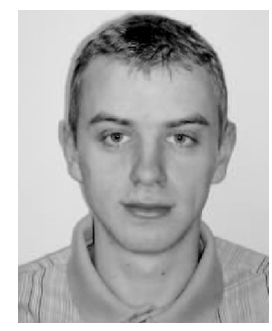

Dirk Deschrijver was born in Tielt, Belgium, on September 26, 1981. He received the Master degree (licentiaat) and Ph.D. degree in computer science from the University of Antwerp, Antwerp, Belgium, in 2003 and 2007, respectively.

From May to October 2005, he was a Marie Curie Fellow with the Scientific Computing Group, Eindhoven University of Technology, Eindhoven, The Netherlands. He is currently a Post-Doctoral Fellow with the Department of Information Technology (INTEC), Ghent University, Gent, Belgium. His research interests include robust parametric macromodeling, rational least squares approximation, orthonormal rational functions, system identification, and broadband macromodeling techniques.

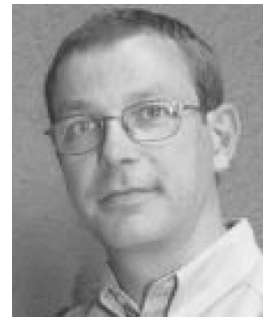

Tom Dhaene (M'94-SM'05) was born in Deinze, Belgium, on June 25, 1966. He received the Ph.D. degree in electrotechnical engineering from the University of Ghent, Gent, Belgium, in 1993.

From 1989 to 1993, he was Research Assistant with the Department of Information Technology, University of Ghent, where his research focused on different aspects of full-wave EM circuit modeling, transient simulation, and time-domain characterization of high-frequency and high-speed interconnections. In 1993, he joined the electronic design automation (EDA) company Alphabit (now part of Agilent). He was one of the key developers of the planar EM simulator ADS Momentum. Since September 2000, he has been a Professor with the Department of Mathematics and Computer Science, University of Antwerp, Antwerp, Belgium. Since October 2007, he has been a Full Professor with the Department of Information Technology (INTEC), Ghent University. He has authored or coauthored over 100 peer-reviewed papers and abstracts in international conference proceedings, journals, and books. He holds two U.S. patents.

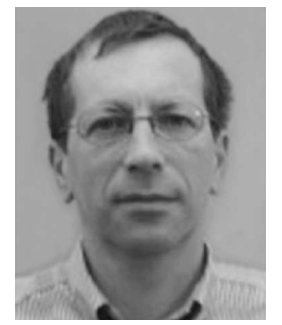

Daniël De Zutter (M'92-SM'96-F'01) was born in 1953. He received the M.Sc. degree in electrical engineering, $\mathrm{Ph} . \mathrm{D}$. degree, and a thesis leading to a degree equivalent to the French Aggrégation or the German Habilitation from Ghent University, Gent, Belgium, in 1976, 1981, and 1984, respectively.

From 1984 to 1996, he was with the National Fund for Scientific Research of Belgium. He is currently a Full Professor of electromagnetics with Ghent University. Most of his earlier scientific work concerned the electrodynamics of moving media. His research currently focuses on all aspects of circuit and EM modeling of high-speed and high-frequency interconnections and packaging, EM compatibility, and numerical solutions of Maxwell's equations. He has authored or coauthored over 140 international journal papers and 150 papers in conference proceedings.

Prof. De Zutter is an associate editor for the IEEE TRANSACTIONS ON MicrowaVe THEORY AND TeChNIQUES. He was a corecipient of the 1995 IEEE Microwave Prize Award presented by the IEEE Microwave Theory and Techniques Society (IEEE MTT-S). He was the recipient of the 1999 Transactions Prize Paper Award presented by the IEEE Electromagnetic Compatibility (EMC) Society. 